\title{
Poster abstracts of the 4th triennial meeting of the European Federation of Conservative Dentistry (EFCD) held in association with the 17th annual meeting of the Spanish Society of Conservative Dentistry (SEOC), 12-14 march 2009, Seville, Spain
}

\author{
Jose C. de la Macorra $\cdot$ Rafael Llamas
}

Published online: 21 January 2009

(C) Springer-Verlag 2009

The 4th triennial meeting of the European Federation of Conservative Dentistry (EFCD) - an event held in association with the 17th annual meeting of the Spanish Society of Conservative Dentistry (SEOC), with concurrent meetings of member associations of EFCD (AODES, DGZ and SIDOC) is the eagerly awaited 2009 ConsEuro: Across European Borders - Prevention, Restoration and Aesthetics. This three day event, in common with previous ConsEuro meetings is a celebration of Conservative Dentistry in Europe.

The first of the three days of the meeting comprises meetings of member organisations of EFCD, poster presentations and precongress workshops. The EFCD members organisations holding meetings as part of 2009 ConsEuro include AODES, DGZ and SIDOC. The poster presentations - a major feature of ConsEuro meetings, are organised in six sessions: four for research - prevention, operative dentistry, endodontics and biomaterials, and two for clinical case presentations and student's presentations. Poster presenters entered into the poster presentation prize

\footnotetext{
J. C. de la Macorra $(\square)$

President of EFCD,

President of the Scientific Committee of the ConsEuro09 meeting,

Madrid, Spain

e-mail: macorra@odon.ucm.es

R. Llamas

President of the ConsEuro09 meeting,

Seville, Spain

e-mail: rllamas@us.es
}

competitions are eligible to win prestigious 2009 ConsEuro prizes. The precongress workshops are on CAD-CAM ceramics, rotary instrumentation in endodontics, clinical utilities, basic periodontal interventions and on the ways to succeed with new, innovative composite systems. Other attractions include a free of charge aesthetic dentistry course for students attending the meeting, a symposium on new criteria for the clinical evaluation of dental restorations and a roundtable discussion on laboratory investigations of adhesive interfaces. The first day of the meeting will conclude with a ceremony to include the award of the 2007 and 2008 EFCD Awards of Excellence to Professor Edwina Kidd (2007) and Professor Erik Asmussen (2008) in recognition of their outstanding contributions to Conservative Dentistry.

The second day of 2009 ConsEuro is dedicated to preventive and operative dentistry, with two simultaneous keynote lecture programmes. The topics covered include fluorides, erosion, the caries process, the intraoral repair of porcelain restorations, periodontal intervention, adhesive technologies and direct and indirect restorations.

The third day of the 2009 ConsEuro meeting comprises roundtable discussions on the restoration of endodontically treated teeth and bleaching and on presentations on modern approaches to root canal therapy and endodontic materials.

Subsequent to 2009 ConsEuro, the EFCD will hold ConsEuro events every two years, with the next ConsEuro meeting (2011 ConsEuro) being held in Istanbul. The EFCD is delighted that the level of support and interest in ConsEuro meetings supports a move to biannual rather than triennial meetings. 